\title{
Human Uterus Transplantation: Have We Opened a Pandora's Box?
}

\author{
Allahbadia Gautam N.
}

Published online: 20 February 2015

(C) Federation of Obstetric \& Gynecological Societies of India 2015

\begin{abstract}
About the Author

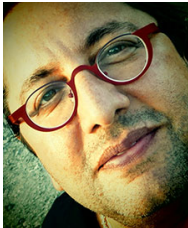

Gautam N. Allahbadia is the Editor-in-chief of the Journal of Obstetrics \& Gynecology of India as well as the IVF Lite (Journal of Minimal Stimulation IVF). He is the Medical Director of Rotunda-The Center for Human Reproduction, the world-renowned fertility clinic at Bandra, Mumbai, India and also the New Hope IVF Clinic at Sharjah, UAE. He is a noted world authority on Ultrasound-guided Embryo Transfers and is one of the pioneers in Third Party Reproduction in SouthEast Asia. He was responsible for India's first trans-ethnic surrogate pregnancy involving a Chinese couple's baby delivered by an unrelated Indian surrogate mother. He has over 100 peer-reviewed publications to his credit and is on the Editorial Board of several International Journals. Throughout his career, he has been instrumental in developing new fertilityat www.gautamallahbadia.com. enhancing protocols and propagating the use of ultrasound in embryo transfer procedures. One can read more about his work
\end{abstract}

According to Greek legend, the first woman, Pandora, was actually sent as a curse to Zeus and was given a present upon her marriage. The present was a box that she was told never to open. Needless to say, her curiosity got the better of her (like eating forbidden fruit) and she unleashed eight demons unto the world [1]. The first seven being the seven deadly sins, and the last, which she managed to capture, was hope. Today, much like Christianity's idea of biting the forbidden fruit, opening Pandora's box refers to getting into a situation over which one has very little control....

Allahbadia G. N. ( $)$, Medical Director

Rotunda-The Center for Human Reproduction, Bandra,

Mumbai, India

e-mail: ivfwaladoc@gmail.com

\section{Introduction}

The only untreatable subgroup of female infertility is absolute uterine factor infertility (AUFI), which is due to congenital or surgical absence of a uterus or presence of a non-functional uterus. The solitary option for a woman with AUFI to become a biological mother today is through a gestational surrogacy, a procedure that is outlawed in many countries globally. Human uterus transplantation is a potential future treatment of AUFI. Akar et al. from Turkey reported the first clinical pregnancy in 2013 after uterus transplantation in a 23-year-old Mayer-Rokitansky-KusterHauser syndrome patient with previous vaginal reconstruction and uterus transplantation [2]. 18 months after the transplant, the endometrium was prepared for transfer of the thawed embryos. The first ET cycle with a single, day-3 thawed embryo resulted in a biochemical pregnancy. The second ET cycle resulted in a clinical pregnancy confirmed with transvaginal ultrasound visualization of an intrauterine 
gestational sac with decidualization. This pregnancy unfortunately did not grow further. Uterine transplantation requires extensive evaluation of the recipient and donor by an experienced multidisciplinary transplantation team both preand post-operatively [3].

Eleven human uterine transplantation attempts had been done worldwide but no live birth had yet been reported till 2014. In 2013, a 35-year-old woman with congenital absence of the uterus (MRKH syndrome) underwent transplantation of the uterus in Sahlgrenska University Hospital, Gothenburg, Sweden [4]. The uterus was donated from a living, 61-year-old, parous donor. In vitro fertilization treatment of the recipient had been done before transplantation, from which 11 embryos were vitrified. The recipient and the donor had essentially uneventful postoperative recoveries. The recipient's first menstruation occurred 43 days after transplantation, and she continued to menstruate at median intervals of 32 days. 1 year after transplantation, the recipient underwent her first single embryo transfer, which resulted in pregnancy. She was then given triple immunosuppression (Tacrolimus, Azathioprine, and Corticosteroids), which was continued throughout pregnancy. She had three episodes of mild rejection, one of which occurred during pregnancy. These episodes were all reversed by corticosteroid treatment. Fetal growth parameters and blood flows of the uterine arteries and umbilical cord were normal throughout pregnancy. The patient was admitted with pre-eclampsia at 31 full weeks and 5 days, and $16 \mathrm{~h}$ later a cesarean section was done because of an abnormal cardiotocogram. A male child with a normal birth weight for gestational age (1775 grams) and with APGAR scores 9, 9, and 10 was born. Brännström et al. thus described the first live birth after human uterus transplantation [4].

\section{Discussion}

Johannesson et al. subsequently reported the 12-month outcome of seven patients with viable uteri after uterus transplantation [5]. Pre-determined immunosuppression was with Tacrolimus and Mychophenolate Mofetil (MMF) for 6 months, and thereafter MMF was withdrawn. Frequent ultrasound examinations were performed to assess uterine appearance and uterine artery blood flow. Cervical biopsies (for histological detection of rejection) were obtained at preset time points, with temporary adjustments of immunosuppression if there were signs of rejection. Menstruations were systematically recorded. All patients showed regular menses after 1-2 months. Uterine artery blood flow was unchanged, with a median pulsatility index of 1.9 (range, 0.5-5.4). Blood levels of Tacrolimus were approximately 10,9 , and $8(\mu \mathrm{g} / \mathrm{L})$ during months 2,9 , and
12, respectively. Four recipients showed mild inflammation in biopsies after MMF withdrawal and were treated with Corticosteroids and Azathioprine during the remainder of the 12 months. Subclinical rejection episodes were detected on ectocervical biopsies in five recipients. Histology showed apoptotic bodies and occasional spongiosis in the squamous epithelium. Moderate infiltration of lymphocytes and neutrophils was seen in the epithelial/stromal interface. All rejection episodes were successfully treated for 2 weeks with Corticosteroids or dose increments of Tacrolimus. The authors demonstrated long-term uterine viability after uterine transplantation, with continued menstruation and unaltered uterine artery blood flow. Subclinical rejection episodes were effectively reversed by temporary increase of immunosuppression [5].

The same Swedish group reported the 6-month results of the first clinical uterus transplantation trial [6]. Durations of donor and recipient surgery ranged from 10 to $13 \mathrm{~h}$ and from 4 to $6 \mathrm{~h}$, respectively. No immediate peri-operative complications occurred in any of the recipients. After 6 months, seven uteri remained viable with regular menses. Mild rejection episodes occurred in four of these patients. These rejection episodes were effectively reversed by Corticosteroid boluses. The two graft losses were because of acute bilateral thrombotic uterine artery occlusions and persistent intrauterine infection. These results demonstrated the feasibility of live donor uterine transplantation with a low-dose immunosuppressive protocol [6].

Johannesson et al. recently summarized the primate uterine transplantation experiments that have paved the way for the human uterine transplantation cases and also addressed the ethics around uterine transplantation [7]. Organ transplantation is no longer reserved for those with a life-threatening disease and neither is organ transplantation together with concurrent immunosuppression a contraindication for pregnancy [8]. The science and research that led to the Swedish team's success evolved over years from work done in basic primate labs to gynecology operating rooms.

Akar et al. from Turkey had demonstrated that uterus retrieval in cadavers with the attached vasculature could be successfully achieved in four of the presented cases [9]. They concluded that uterus retrieval with its vasculature and supporting sacro-uterine, vesico-uterine peritoneal folds is an anatomically feasible procedure in preparation for uterus transplantation.

Gauthier et al. described the feasibility of human uterus retrieval after donation after brain death [10]. A specific organ retrieval procedure was performed. At the end of the procedure, the uterus was removed together with the hypogastric vessels, parametria, and vaginal fornix. The tolerance of the uterus to cold ischemia was evaluated with histology and TUNEL reaction up to $24 \mathrm{~h}$. Between August 
1, 2012 and July 31, 2013, seven uteri were retrieved from 14 female multiorgan donors. The hypogastric vessels could be preserved in all cases but for one vein loss in the first retrieval. Histology studies did not find major morphologic changes after $24 \mathrm{~h}$ of cold ischemia. Apoptosis was rare. The paper concluded that uterine retrieval could be part of a reproducible multiorgan procurement procedure and seems readily acceptable to the families of the deceased donor [10].

Saso et al. investigated, developed, and evaluated anatomical, surgical, and anastomotic aspects necessary for a successful uterine transplant in a large animal model [11]. Five auto-transplants were performed in five ewes of proven fertility. One procedure was abandoned because of the inappropriate size of sheep model. Another procedure was halted because the animal suffered from respiratory failure in the immediate intra-operative period. Three transplants were completed. In those, at least two of the four possible anastomoses were finished, and the grafted uteri demonstrated immediate perfusion and appropriate viability 45 min post-operatively. The group concluded that internal to external iliac vessel anastomoses are an acceptable surgical technique that should be applied in a human model to ensure adequate subsequent uterine perfusion [11].

Wang et al. evaluated the surgical feasibility of uterus autologous transplantation in female cynomolgus monkeys and explored the effect of microsurgical techniques [12].From May 2011 to March 2014, six female cynomolgus monkeys, aged 7-12 years, were randomly divided into two surgical groups. In group A, surgeries were performed with naked eyes. In group B, uterus re-transplantation was performed under $10 \times$ microscopy. Anatomical data and operative durations were recorded and analyzed. Viable uterine tissue and vascular patency were observed on trans-abdominal ultrasonography and second-look laparotomy after 3 months. This study demonstrated the feasibility of uterus transplantation by vascular anastomosis in cynomolgus monkeys. And assistance of microsurgical technique can significantly improve the success rate of arterial anastomosis during uterus transplantation [12].

Uterine transplantation studies have been performed in many animals, and accumulation of data from these studies has brought uterus transplantation within reach of routine clinical application. However, more basic studies in primates are needed to fine-tune the surgical and post-operative skills, and data from research in primates can provide important information for establishment of uterus transplantation in humans [13].

A recent publication aimed to assess whether fertility was possible following allogeneic uterus transplantation, when the recipient had demonstrated long-term survival and had been administered immunosuppression [14]. Nine allogeneic uterus transplantations in New Zealand White rabbits were performed using a pre-determined protocol. Tacrolimus was the immunosuppressant selected. Pregnancy was achieved after rabbit allogeneic uterine transplantation, but serial ultrasounds suggested that fetal demise occurred prior to scheduled necropsy. This study represents only the third example of conception and pregnancy following an animal allogeneic uterine transplant [14].

Gauthier et al. sent anonymous questionnaires to associations of patients with Mayer-Rokitansky-Küster-Hauser (MRKH) syndrome and to women suffering from AUFI devised to sound out their attitude toward uterine transplantation and toward adoption and gestational surrogacy [15]. Sixty patients answered the questionnaire. 35 patients were willing to take part in a clinical study involving uterine transplantation despite the uncertainty of the outcome and the potential risks involved. Gauthier's team summarized that because of the feelings of vulnerability engendered by AUFI, any uterus transplantation program should provide full information to patients and ensure they are carefully screened and selected.

\section{Conclusions}

Uterus transplantation is the first available treatment for absolute uterine infertility, which is caused by the absence of the uterus or presence of a non-functional uterus. After extensive animal research, also involving non-human primates, a small number of human uterine transplantation cases have, over the past 5 years, been performed in Saudi Arabia, Turkey and Sweden. Uterus transplantation involves four parties-recipient, donor, partner of the recipient, and the future child-and is a subject of ethical controversies. Murphy in a recent bioethics publication [16] asked a pertinent question "Should this kind of transplantation prove routine and effective for those women, would there be any morally significant reason why men or transgender women should not be eligible for the same opportunity for gestation?" There was hope for the women suffering from AUFI when the first baby was delivered, but now the Pandora's box could throw up many more surprises that we never dreamt of.

\section{References}

1. Lamberton R. Hesiod, Chapter II, "The Theogony", and Chapter III, "The Works and Days". New Haven: Yale University Press, ISBN 0-300-04068-7. 1988, pp. 96-103.

2. Akar ME, Ozkan O, Aydinuraz B, et al. Clinical pregnancy after uterus transplantation. Fertil Steril. 2013;100(5):1358-63. doi: 10.1016/j.fertnstert.2013.06.027.

3. Ozkan O, Akar ME, Erdogan O, et al. Uterus transplantation from a deceased donor. Fertil Steril. 2013;100(6):e41. doi: 10.1016/j.fertnstert.2013.06.041. 
4. Brännström M, Johannesson L, Bokström $\mathrm{H}$ et al. Livebirth after uterus transplantation. Lancet. 2014. doi:10.1016/S01406736(14)61728-1.

5. Johannesson L, Kvarnström N, Mölne J, et al. Uterus transplantation trial: 1-year outcome. Fertil Steril. 2015;103(1):199-204. doi:10.1016/j.fertnstert.2014.09.024.

6. Brännström M, Johannesson L, Dahm-Kähler P, et al. First clinical uterus transplantation trial: a six-month report. Fertil Steril. 2014;101(5):1228-36. doi:10.1016/j.fertnstert.2014.02.024.

7. Johannesson L, Dahm-Kähler P, Eklind S, et al. The future of human uterus transplantation. Womens Health (Lond Engl). 2014;10(4):455-67. doi:10.2217/whe.14.22.

8. Johannesson L, Enskog A. Experimental uterus transplantation. Best Pract Res Clin Obstet Gynaecol. 2014;28(8):1198-210. doi: 10.1016/j.bpobgyn.2014.07.018.

9. Akar ME, Ozkan O, Ozekinci M, et al. Uterus retrieval in cadaver: technical aspects. Clin Exp Obstet Gynecol. 2014;41(3): 293-5.

10. Gauthier T, Piver P, Pichon N, et al. Uterus retrieval process from brain dead donors. Fertil Steril. 2014;102(2):476-82. doi: 10.1016/j.fertnstert.2014.04.016.
11. Saso S, Petts G, Thum $M$ et al. Achieving uterine auto-transplantation in a sheep model using iliac vessel anastomosis: a short-term viability study. Acta Obstet Gynecol Scand. 2014. doi: 10.1111/aogs.12550.

12. Wang Y, Zhu Y, Yu P, et al. Uterine autologous transplantation in cynomolgus monkeys: a preliminary report of 6 cases. Zhonghua Yi Xue Za Zhi. 2014;94(47):3774-7.

13. Kisu I, Banno K, Mihara M, et al. Uterine transplantation in primates: a mini-review of the literature. Transplant Proc. 2014;46(4):1212-6. doi:10.1016/j.transproceed.2013.10.046.

14. Saso S, Petts G, David AL, et al. Achieving an early pregnancy following allogeneic uterine transplantation in a rabbit model. Eur J Obstet Gynecol Reprod Biol. 2015;185:164-9. doi: 10.1016/j.ejogrb.2014.12.017.

15. Gauthier T, Garnault D, Therme JF, et al. Uterine transplantation: Is there a real demand? Gynecol Obstet Fertil. 2015; doi: 10.1016/j.gyobfe.2014.12.005.

16. Murphy TF. Assisted gestation and transgender women. Bioethics. 2014. doi: 10.1111/bioe.12132. 